\title{
Linfomas asociados a infección por virus de inmunodeficiencia humana en un complejo hospitalario de la Región Metropolitana, Chile: 1990-2002. Reporte de 14 casos y revisión
}

\author{
Guido Osorio S. y Cristián Montenegro U.
}

\section{Lymphomas and HIV infection in a reference hospital of Santiago, Chile: 1990-2002: Report of 14 cases and review}

The association of HIV infection and lymphoma in patients attending at the South Health Metropolitan Reference Centre is presented. Objective: to analyse its incidence, clinical and pathologic manifestations, treatment and outcome. Period of study: January 1990 to December 2002. Results: 14 cases were detected, 10 non Hodgkin lymphoma patients (7 with high malignancy and $50 \%$ in stages III-IVB) and 4 with Hodgkin lymphoma ( 3 with mixed cellularity, 2 in stage IVB). The annual incidence was $0.68 \%$. Ten patients were classified under stage C3 of AIDS CDC criteria, the mean CD4 count was 139 cells $/ \mathrm{mm}^{3}$ and mean CV was 5,32 log. Eighty six percent of patients presented with unique or multiples lymphonodes, with predominance of advanced lymphoma stage. Conventional CHOP chemotherapy was the treatment for high risk and extended non Hodgkin lymphomas and for extended Hodgkin lymphomas the ABVD protocol was administered. Six patients received antiretroviral therapy, 4 simultaneously with chemotherapy. Global mortality in this series was $71 \%$, attributable to tumor disease per se or to sepsis. Four patients survived (18 to 50 months) in complete remission, 2 non Hodgkin lymphomas and 2 Hodgkin lymphomas. The low incidence of lymphoma and AIDS association and the high frequency of lymphomas with localized or generalized lymphonodes in this series are remarkable.

Key words: lymphoma, AIDS, clinical, histopathology, immunology, treatment, evolution.

Palabras claves: linfoma, SIDA, clínica, histopatología, inmunología, tratamiento, evolución.

\section{Introducción}

$\mathrm{E}$ n Chile, la asociación de SIDA y linfoma aparece descrita en dos series de pacientes con infección por VIH publicadas en 1995 y 2001. Un centro de atención integral de enfermedad por VIH de Santiago de Chile comunicó una casuística de 240 pacientes infectados por VIH, entre 1988 y 1993, describiendo cuatro linfomas no Hodgkin (LNH) ${ }^{1}$. En otra publicación posterior del mismo grupo, en 316 pacientes seropositivos, acumulados desde noviembre de 1996 a marzo de 1999, encontraron tres casos de la asociación con linfoma².

Según datos de la Comisión Nacional del SIDA (CONASIDA), a mayo de 2004, la incidencia acumulada de LNH era de 26 casos en 3.218 pacientes y la prevalencia de $0,8 \%{ }^{3}$.

Interesados en sistematizar nuestra experiencia acerca de esta asociación de enfermedades, se diseñó un estudio para conocer la situación en el Servicio de Salud Metropolitano Sur (SSMS) de los pacientes que presentan linfoma e infección por VIH; su incidencia, sintomatología y signología que orienten al diagnóstico; su extensión, el tipo histológico, grado de inmunosupresión, carga viral (CV) y la respuesta a los tratamientos efectuados.

\section{Pacientes y Método}

Estudio retrospectivo y descriptivo. Los pacientes se identificaron mediante la búsqueda en las siguientes fuentes:

- Registros estadísticos denominados "hoja de registro semanal de ingresos y altas" del Hospital de Enfermedades Infecciosas Dr. Lucio Córdova (HDLC);

- Las fichas clínicas exclusivas e independientes de la historia clínica del hospital, de pacientes con linfomas, vivos y fallecidos, del Departamento de Hematología del Hospital Barros Luco Trudeau (HBLT);
Universidad de Chile y Hospital Barros Luco Trudeau, Santiago, Chile

Servicio de Medicina (GOS) Hospital Enfermedades Infecciosas Prof. Dr. Lucio Córdova (CMU)

Recibido: 16 mayo 2006 Aceptado: 20 octubre 2006

Correspondencia a: Guido Osorio S. gosorios77@hotmail.com 
- Registro estadístico anual por fecha, nombre, diagnóstico y otros datos, elaborado por el Departamento de Estadísticas del SSMS.

Se incorporaron al estudio todos los pacientes con la asociación diagnóstica de linfoma y SIDA, atendi-

\section{Tabla 1. Clasificación histológica del panel de expertos para los linfoma no Hodgkin (Working Formulation) (ref 4)}

De bajo grado

A. Linfoma maligno linfocítico pequeño

B. Linfoma maligno folicular de células pequeñas hendidas predominantes: - áreas difusas - esclerosis

C. Linfoma maligno folicular mixto de células pequeñas hendidas y grandes: áreas difusas - esclerosis

De grado intermedio

A. Linfoma maligno folicular de células grandes predominantes: - áreas difusas - esclerosis

B. Linfoma maligno difuso de células pequeñas hendidas

C. Linfoma maligno difuso mixto, células pequeñas y grandes: - esclerosis componente epitelioide

D. Linfoma maligno difuso de células grandes:

- hendidas

- no hendidas

esclerosis

De alto grado

A. Linfoma maligno de células grandes, inmunoblástico: -plasmocitoide - de células claras - polimorfo - componente epitelioide

B. Linfoma maligno linfoblástico: - células convolutadas - células no convolutadas

C. Linfoma maligno de células no hendidas: - Burkitt áreas foliculares dos en el HDLC y el Departamento de Hematología del HBLT, entre enero de 1990 y diciembre de 2002. No hubo exclusiones. Se confeccionó un formulario especial en el que se recogieron los siguientes datos: edad, estado civil, fecha de diagnóstico de infección por $\mathrm{VIH}$, forma de contagio y antecedentes mórbidos. Respecto de la infección por VIH se registraron: etapa de la clasificación $\mathrm{CDC}$ al momento del diagnóstico, recuento absoluto de linfocitos T CD4 (céls $\left./ \mathrm{mm}^{3}\right), \mathrm{CV}$ (copias/ml de ARN), infecciones oportunistas previas al diagnóstico de la asociación de ambas enfermedades, serología para Toxoplasma gondii, baciloscopias, infecciones de transmisión sexual, medicamentos utilizados en el tratamiento de estas infecciones, incluyendo el TARV, y con respecto a la enfermedad oncológica: fecha del diagnóstico de linfoma, síntomas sistémicos y signos iniciales, exámenes de laboratorio (hemograma, velocidad de eritrosedimentación (VHS), estudio de hemostasia, perfil bioquímico, deshidrogenasa láctica (LDH), $\beta$-2 microglobulinemia, estudio de LCR, imagenología, procedimientos diagnósticos diversos, resultados de biopsia, evolución clínica y de laboratorio, tratamiento efectuado para el linfoma, complicaciones y sobrevida.

El diagnóstico de infección por VIH fue confirmado con las técnicas de inmunofluorescencia indirecta (IFI) en el Instituto de Salud Pública (ISP) y el de linfoma mediante estudios histológicos. La tipificación histológica de los LNH se hizo por la clasificación Working Formulation (Tabla 1) y la de linfoma de Hodgkin (LH) por la Clasificación de Rye modificada. El estadio clínico se estableció según criterios de Ann Arbor (Tabla 2) . El estudio de extensión, la evaluación del estadio clínico, el tratamiento y la respuesta clínica a quimioterapia o radioterapia, se efectuaron de acuerdo con el Protocolo del Programa Adultos Nacional de Drogas Antineoplásicas (PANDA) para linfomas, vigente a la fecha del diagnóstico. Se consideró remisión completa (RC) a la desaparición de cualquier evidencia de enfermedad valorable al finalizar la tera-

\section{Tabla 2. Clasificación de Ann Arbor que permite definir etapa evolutiva de los linfomas (ref 5)}

Estadio I. Compromiso de un ganglio o grupo ganglionar único (I) o compromiso localizado de una región extraganglionar (IE)

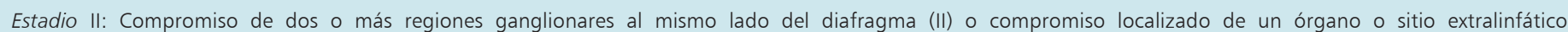
asociado y sus ganglios linfáticos regionales, con o sin compromiso de otras regiones ganglionares al mismo lado del diafragma (IIE)

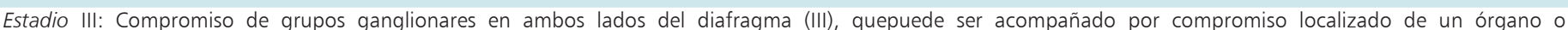
sitio extralinfático asociado (IIIE) o por compromiso esplénico (IIIS) o ambos (IIIES)

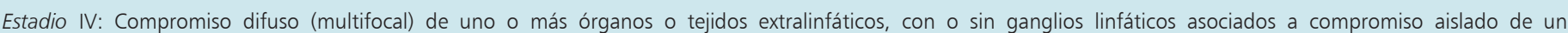

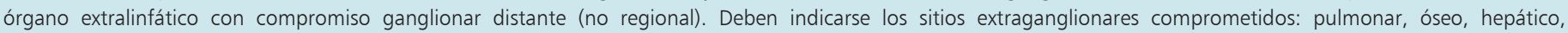
cerebro, médula ósea, pleura, piel, ojos, otros 
pia; remisión parcial (RP) a cualquier disminución de la masa tumoral valorable sin alcanzar $\mathrm{RC}$; y no respuesta (NR) a la progresión de la enfermedad o ausencia de mejoría durante el tratamiento.

\section{Resultados}

Se detectaron 14 pacientes, 12 varones y dos mujeres. En la Figura 1 se presentan los pacientes con infección por VIH diagnosticados durante el período 1990-2002 en el área geográfica del Servicio de Salud Metropolitano Sur; se presentan, además, los pacientes con la asociación linfoma-infección por VIH, por año, y la incidencia anual de esta asociación.

Las edades correspondieron a un rango de 26 a 49 años, con una edad promedio de 35,5 años \pm 2 DS. En todos los pacientes, la adquisición del VIH fue por contacto sexual. Sólo en tres de los 12 pacientes se hizo el diagnóstico de linfoma antes de que se conociera su condición de infectado por VIH. En la Tabla 3 se detalla la frecuencia y el porcentaje de presentación de los principales síntomas, signos, carga viral y recuento de linfocitos CD4, en linfomas asociados a VIH.

En la forma de presentación de los linfomas es destacable una frecuencia claramente mayor de adenopatías que de los otros signos iniciales (12 de 14 pacientes), seguido de una tumoración que se presentó en cinco de 13 pacientes (Tabla 3). La localización extranodal exclusiva se dio en un caso de tumor cerebral y un tumor de seno maxilar.

En dos de los 14 pacientes no se logró obtener el recuento de $\mathrm{CD} 4 \mathrm{y}$ en cuatro no se conoció su $\mathrm{CV}$, debido a que estos pacientes presentaron la infección por VIH asociada a linfoma antes de 1997, fecha en la que se comenzó a solicitar en forma rutinaria la CV y CD4 para definir etapa CDC y en el seguimiento de estos pacientes. Sólo cuatro de los 14 pacientes tenían $\mathrm{CV}>$ 100.000 copias de ARN/ml con CD4 menores a 100 células $/ \mathrm{mm}^{3}$.

La mayoría de los pacientes fueron clasificados en etapa clínica C3 de SIDA $(\mathrm{n}=10)$, en el momento del diagnóstico de su infección por VIH; dos en $\mathrm{C} 2$; uno en B3 y otro en A2.

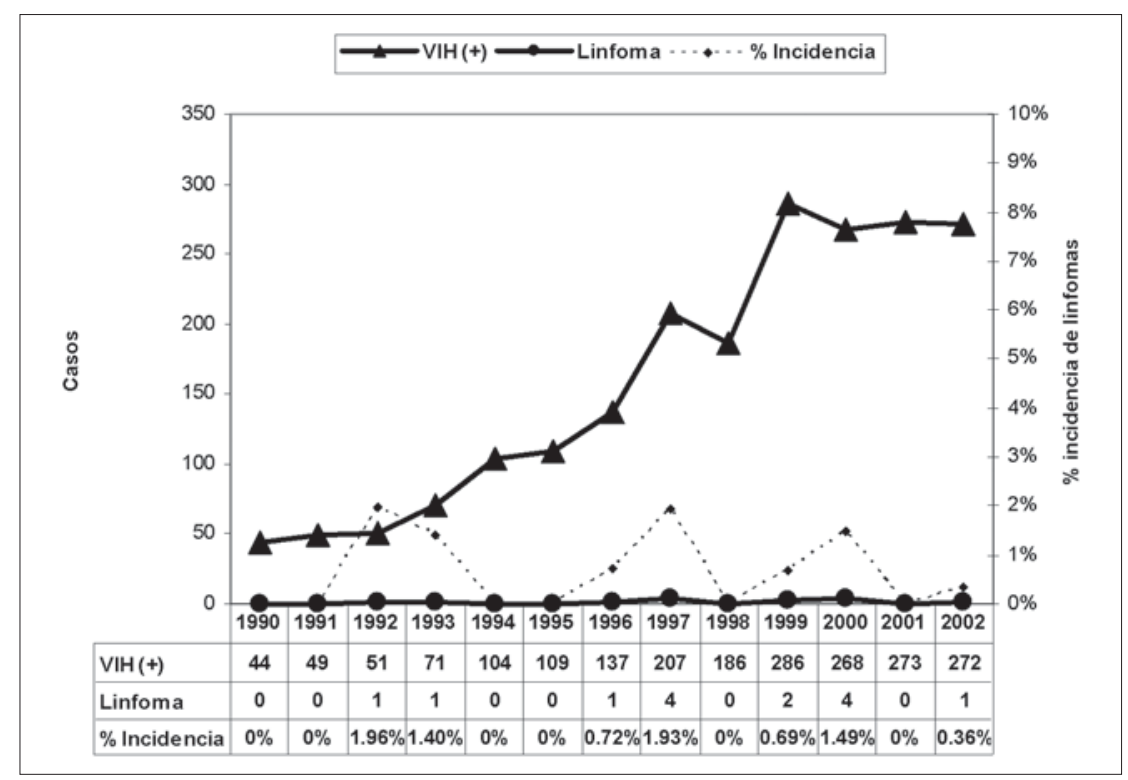

Figura 1. Número de casos de infección por VIH y linfoma en la población del Servicio de Salud Metropolitano Sur. 1990-2000.

\section{Tabla 3. Manifestaciones clínicas, carga viral y recuento de linfocitos T CD4, en linfomas asociados a la infección por VIH}

\begin{tabular}{|c|c|c|c|c|}
\hline Síntomas y signos & $\mathbf{n}$ & $\%$ & Rango & Promedio \\
\hline Sensación febril & 4 & 28,5 & - & - \\
\hline Sudoración & 4 & 28,5 & - & - \\
\hline Baja de peso & 3 & 21,4 & - & - \\
\hline Compromiso de estado general & 5 & 35,7 & - & - \\
\hline Ictericia & 1 & 7,1 & - & - \\
\hline Síntomas neurológicos & 3 & 21,4 & - & - \\
\hline Palidez & 3 & 21,4 & - & - \\
\hline Hepato-esplenomegalia & 4 & 28,5 & - & - \\
\hline Fiebre & 3 & 21,4 & - & - \\
\hline Tumoración & 5 & 35,7 & - & - \\
\hline Adenopatías & 13 & 92,8 & - & - \\
\hline Focalización neurológica & 2 & 14,2 & - & - \\
\hline Carga viral (copias ARN/ml) & & & $24.000-1.100 .000$ & 212.600 \\
\hline CD4 (céls/mm³) & & & $20-274$ & 139,5 \\
\hline
\end{tabular}


Seis pacientes recibieron TARV. Las respuestas al TARV fueron variables; dos pacientes - 1 LNH IB-B y 1 LH IVB etapa C3, con CD4 de 20 y 45 céls $/ \mathrm{mm}^{3}$, CV de 440.000 y 1.100 .000 copias ARN/ml- no respondieron y fallecieron. Los cuatro pacientes restantes sobreviven al momento de este análisis y corresponden a: $2 \mathrm{LNH}$ localizados I-E y IA, con CD4 de 100 y 193 céls $/ \mathrm{mm}^{3}$ y $\mathrm{CV}$ de 150.000 y 99.000 copias ARN/ml respectivamente; y dos pacientes con LH de celularidad mixta, estadios II-A y III SA, con recuentos de CD4 de 274, 256 céls $/ \mathrm{mm}^{3}$ y CV de 30.000 y 34.000 copias ARN/ml.

Cuatro pacientes recibieron simultáneamente TARV y el tratamiento de su linfoma, tres de ellos están en RC y vivos. Dos pacientes fueron tratados con TARV post quimioterapia del linfoma, uno de ellos permanece vivo y en RC. Seis pacientes presentaron complicaciones infecciosas durante el tratamiento: infección urinaria por Escherichia coli multiresistente; neumonía intrahospitalaria; traqueobronquitis aguda y candidiasis orofaríngea; dos casos candidiasis oral y otro, neumopatía aguda lobar. Dos enfermos fallecieron a causa de sepsis no controlada y uno por neumopatía aguda.

En lo que respecta a los exámenes de laboratorio, (Tabla 4), el hemograma no reveló algún patrón característico que permitiera orientar el diagnóstico. La hemoglobina estaba dentro de rangos normales en siete enfermos y en un número similar existía anemia, en dos

Tabla 4. Valores promedios de exámenes de laboratorio en linfomas asociados a SIDA

\begin{tabular}{|c|c|c|c|c|c|}
\hline Paciente & Hemoglobina (g) & Leucocitos $/ \mathrm{mm}^{3}$ & LDH $(\mathrm{mg} / \mathrm{dL})$ & Bilirrubinemia total $(\mathrm{mg} / \mathrm{dL})$ & $\beta-2$ microglobulina (mg/L) \\
\hline Promedio & $11,3 \pm 2 \mathrm{DS}$ & $4.700 \pm 2 D S$ & $393,4 \pm 2 D S$ & $1,53 \pm 2 \mathrm{DS}$ & $4,46 \pm 4 \mathrm{DS}$ \\
\hline
\end{tabular}

\begin{tabular}{|c|c|c|c|c|c|c|c|}
\hline $\begin{array}{l}\text { Paciente } \\
\text { n }\end{array}$ & $\begin{array}{l}\text { Diagnóstico por biopsia ganglionar, } \\
\text { BMO u otra* }\end{array}$ & $\begin{array}{l}\text { Estadio del } \\
\text { linfoma }\end{array}$ & Tratamiento & $\begin{array}{l}\text { Tipo de } \\
\text { respuesta } \\
\text { terapéutica }\end{array}$ & $\begin{array}{l}\text { Causa de } \\
\text { deceso }\end{array}$ & $\mathbf{F} / \mathbf{V}$ & $\begin{array}{l}\text { Sobrevida } \\
\text { (meses) }\end{array}$ \\
\hline 1 & $\begin{array}{l}\text { LNH difuso de células grandes inmunoblástico } \\
\text { plasmocitoide del seno maxilar }\end{array}$ & I-E & $\begin{array}{l}\text { CHOP } \\
\text { Radioterapia }\end{array}$ & $\mathrm{RC}$ & & V & 41 \\
\hline 2 & LNH bajo grado malignidad & III-A & CHOP & NR & Progresión & $\mathrm{F}$ & 27 \\
\hline 3 & LNH inmunoblástico & IV-B & $\mathrm{CHOP}$ & NR & Progresión & $\mathrm{F}$ & 17 \\
\hline 4 & LNH intermedio difuso de células grandes & III-B & $\begin{array}{l}\text { Clorambucil } \\
\text { Prednisona }\end{array}$ & NR & Progresión & $\mathrm{F}$ & 17 \\
\hline 5 & $\begin{array}{l}\text { LNH intermedio de glándula submaxilar*; } \\
\text { BMO: LNH difuso células grandes }\end{array}$ & IV-B & $\mathrm{CHOP}$ & NR & Sepsis & $\mathrm{F}$ & 2 \\
\hline 6 & LNH difuso de células grandes (*biopsia cerebral) & I-E & Radioterapia & NR & Progresión & $\mathrm{F}$ & 13 \\
\hline 7 & LH celularidad mixta; BMO: normal & $\|-A$ & $\begin{array}{l}\text { Radioterapia, } \\
\text { NOVP, ABVD }\end{array}$ & $\mathrm{RC}$ & & V & 25 \\
\hline 8 & LH depleción linfocítica & IV-B & & NR & Progresión & $\mathrm{F}$ & 2 \\
\hline 9 & $\begin{array}{l}\text { LNH cutáneo*, difuso inmunoblástico; } \\
\text { BMO: mielodisplasia }\end{array}$ & IV-B & & NR & Progresión & $\mathrm{F}$ & 1 \\
\hline 10 & LH celularidad mixta; BMO: Normal & III-SA & $\begin{array}{l}\text { Radioterapia } \\
\text { ABVD }\end{array}$ & $\mathrm{RC}$ & & V & 7 \\
\hline 11 & LNH tipo Burkitt & IV-B & $\begin{array}{l}\text { CHOP, } \\
\text { ARA- C intratecal }\end{array}$ & NR & Sepsis & $\mathrm{F}$ & 2 \\
\hline 12 & LH celularidad mixta; BMO: infiltración por LH & IV-B & C-MOPP, CHOP & NR & Progresión & $\mathrm{F}$ & 11 \\
\hline 13 & LNH tipo F; BMO: normal & I-A & CHOP, ESHAP & $R P, R C$ & & V & 5 \\
\hline 14 & LNH tipo G; BMO: hemosiderosis & IV-B & $\mathrm{CHOP}$ & $N R$ & Sepsis & $\mathrm{F}$ & 3 \\
\hline
\end{tabular}


de ellos grave, pero sin caracteres especiales. En ocho pacientes se apreció leucopenia en rangos de 1.400 a 4.400 céls $/ \mathrm{mm}^{3}$. La LDH se elevó sobre $240 \mathrm{mg} / \mathrm{dl}$ en 9 pacientes. En seis casos se registró aumento de $\beta-2$ microglobulinemia sobre $1,9 \mathrm{mg} / \mathrm{L}$.

En once pacientes el diagnóstico se obtuvo por biopsia ganglionar, en un paciente por biopsia de glándula submaxilar, en uno por biopsia de piel y en otro mediante biopsia cerebral. En este último predominaban los síntomas neurológicos y una TAC de cerebro mostraba una imagen redondeada con captación anular de 20 × $20 \mathrm{~mm}$ en la región temporal posteromedial. En seis de los casos se hizo biopsia de médula ósea, encontrándose infiltración en dos de ellos, un LH y otro LNH.

Diez enfermos correspondieron a LNH y cuatro a LH; tres a celularidad mixta y una depleción linfocítica (Tabla 5). Los pacientes fueron evaluados en el grado de extensión del linfoma: siete se encontraban en etapa IV- B, uno en etapa III- B, dos en III-A, uno en II-A, otro I-A y dos en etapa I-E (Tabla 5).

Los esquemas de quimioterapia utilizados en el tratamiento de los linfomas fueron: CHOP (ciclofosfamida, doxorrubicina, vincristina, prednisona); NOVP (mitoxantrona, vincristina, vinblastina, prednisona); ABVD (doxorrubucina, bleomicina, vinblastina, dacarbazina); C-MOPP (ciclofosfamida, vincristina, procarbazina, prednisona) y ESHAP (etoposido, cisplatino, citarabina, metilprednisolona).

Ocho pacientes recibieron tratamiento con quimioterapia $\mathrm{CHOP}$, otro con clorambucil y prednisona; los pacientes con $\mathrm{LH}$ recibieron tratamiento con radioterapia y los esquemas NOVP, ABVD, CHOP y ESHAP (Tabla 5).

Al momento del término de este registro, siete pacientes habían fallecido por progresión del tumor y tres por cuadros sépticos. La mortalidad global de la serie fue de $71 \%$. Sobreviven hasta agosto de 2004: un paciente con LNH difuso inmunoblástico tratado con CHOP y radioterapia (50 meses de sobrevida), una mujer con LH, celularidad mixta IIB que fue tratada con combinación de radioterapia y quimioterapia NOVP y por recidiva $\mathrm{ABVD}$, completó 42 meses de $\mathrm{RC}$, otra mujer con LH, celularidad mixta tratado con radioterapia y esquema $A B V D$, que sobrevive por 18 meses en $\mathrm{RC}$, y un último paciente con $\mathrm{LNH}$ difuso mixto, estadio IA, que después de recibir seis ciclos CHOP recidivó y luego de tres ciclos ESHAP, permanece vivo y en RC durante ya 23 meses.

\section{Discusión}

En el área geográfica Sur de la Región Metropolitana, desde 1986 al año 2002, se han acumulado 2.161 casos de VIH y durante ese lapso sólo se han diagnosticado 14 casos de asociación con linfoma, lo que da una incidencia acumulada de $0,68 \%{ }^{6}$. En otra área de Santiago, en las dos series mencionadas en la introducción, las incidencias oscilan entre 1 y $1,7 \%$.

Estos valores están muy por debajo del 3 a $10 \%$ señalado en la literatura internacional ${ }^{7}$, quizás debido a dificultades en la obtención del diagnóstico de linfoma en su localización extranodal, en especial de ubicación en el SNC. No podemos descartar el traslado espontáneo de algún paciente en control en nuestra institución a otro centro asistencial, como otro factor determinante de una menor incidencia a la descrita. Algunos estudios señalan que la introducción de TARV de alta eficacia elevó la asociación de 10 a $14 \%{ }^{8}$. Otros autores responsabilizan en cambio a la TARV de su disminución, aunque reconocen que falta confirmación para esta hipótesis. Una base internacional de datos de 48.000 individuos infectados por VIH de E.U.A., Europa y Australia, encontró un descenso de 42\% en la incidencia de LNH desde 1997 hasta 1999, en comparación con aquellos desde 1992 a $1996^{9}$.

El diagnóstico de VIH precede al del linfoma en 47 a $57 \%$ de los pacientes ${ }^{10-12}$. En $30 \%$ el diagnóstico de infección por VIH se hace en el momento del diagnóstico del $\mathrm{LNH}^{13}$.

En tres de14 pacientes (21\%), en nuestra casuística, el diagnóstico de linfoma precedió al de la infección por VIH; en ellos el VIH fue detectado dentro de los 10 meses siguientes.

La edad mediana de la muestra fue de 31 años, dentro del rango de 30 a 39 años descrita en la literatura médica ${ }^{8}$.

Sólo dos de los pacientes eran mujeres, lo que se relaciona con la mayor incidencia de infección por VIH observada en hombres en nuestro medio $(88 \% \mathrm{v} / \mathrm{s}$ $12 \%$; razón hombre: mujer $=6,8: 1)^{13,14}$.

Contrastando con lo descrito en la literatura internacional que, en su mayor parte, señala que los $\mathrm{LNH}$ son frecuentemente extranodales diseminados ${ }^{7,11}$, con porcentajes que oscilan entre 40 y $90 \%$, en nuestra casuística la sospecha de diagnóstico de linfoma se basó en la presencia de adenopatías.

Los pacientes con esta asociación se presentan con síntomas sistémicos en 60 a $70 \%$ de los casos. Además, una serie de alteraciones hematológicas: anemia en $75 \%$, leucopenia en $44 \%$, linfopenia en $87 \%$ y trombocitopenia en $30 \%{ }^{15}$. En la muestra estudiada, ni la presencia de síntomas sistémicos ni el hemograma, ayudaron a sospechar el diagnóstico de linfoma.

El LNH primario del SNC es frecuente en pacientes con infección por VIH y se presenta como localización extranodal exclusiva en 25 a $32 \%$ de los casos $^{16}$. En ocasiones, sólo el fracaso del tratamiento empírico 
para toxoplasma, aplicado infructuosamente durante dos semanas, en presencia de TAC o RM compatibles, ha hecho plantear el diagnóstico del linfoma, cuya confirmación debe establecerse con la biopsia cerebral ${ }^{17}$. Todo esto concuerda con el único caso de nuestra casuística que presentó compromiso del SNC. Los pacientes con linfoma cerebral sobreviven entre 2 y 5 meses, el nuestro lo hizo durante 13 meses.

En esta casuística no encontramos correlación respecto de la elevación de LDH y $\beta$-2 microglobulinemia en la medición del pronóstico y diferenciación del linfoma ${ }^{18}$.

El LNH asociado a infección por VIH es un linfoma de células B. Alrededor de 95\% de los LNH en pacientes con SIDA son de malignidad alta o intermedia. En esta casuística predominaron los LNH de alta malignidad. En la literatura científica, $58 \%$ se presenta en estadios IV o IIIB; esto concuerda con lo encontrado en nuestros casos.

El LH se presenta en forma muy agresiva, a menudo con afección extra-ganglionar, cuando se asocia a infección por VIH. Tiene menor frecuencia de compromiso de mediastino, síntomas de categoría B y un recuento medio de CD4 de 300 céls $/ \mathrm{mm}^{3}$ o menos ${ }^{19,20}$. La supervivencia media es de 8 a 20 meses. En $66 \%$ de los pacientes se describe un tipo histológico de celularidad mixta, $82 \%$ se diagnostica en etapa II o IV y $48 \%$ tiene compromiso de la médula ósea. Los cuatro casos de esta muestra coincidieron con la histología más frecuente y la extensión. Se señala que los LNH relacionados con VIH tienen un recuento promedio de CD4 entre 100 y 200 células $/ \mathrm{mm}^{3}{ }^{16}$. Esta situación sólo se dio en tres casos fallecidos.

El tratamiento del linfoma asociado con infección por VIH presenta el reto de integrar la terapia apropiada para el linfoma con las limitaciones impuestas por la infección del $\mathrm{VIH}^{21}$. No existe consenso sobre cuál es la terapia óptima.

Estos pacientes se caracterizan por una mayor susceptibilidad a padecer infecciones oportunistas y una reserva medular muy reducida. Esto dificulta la administración de quimioterapia a dosis plena, comprometiendo su potencial curativo. Se han empleado diversos esquemas, con porcentajes de remisión completa que no superan el $50 \%$, y supervivencias medianas muy abreviadas, de 6 a 9 meses ${ }^{22,23}$, con frecuentes recaídas en el SNC. La tendencia actual -y lo preconiza recientemente el PANDA del MINSAL-, es utilizar el esquema convencional $\mathrm{CHOP}^{24}$ y otros emplean el mBACOD (metotrexato, bleomicina, doxorrubicina, ciclofosfamida, vincristina y dexametasona) ${ }^{25}$, en dosis reducidas, con adición de factores estimuladores de colonias o el esquema de leucemia linfoblástica aguda, sobre todo cuando existe compromiso del SNC o bien, radioterapia total de cerebro. En la casuística predominó el empleo del esquema CHOP en ocho pacientes sin respuestas y supervivencias de dos a 41 meses. En el paciente con compromiso de SNC después de tratar con $\mathrm{CHOP}$, por intolerancia se intentó ara-C intratecal y radioterapia, sin respuesta.

En el LH asociado a infección por VIH se estipula que la respuesta a la terapia sería mejor. En los cuatro casos de esta muestra, uno (IV-B) recibió seis ciclos de C-MOPP con RP y seis ciclos de CHOP y TAR, con supervivencia de 11 meses y fallecimiento al cabo de este lapso. Otro caso (II-A), también con celularidad mixta, recibió radioterapia cervical seguida de esquema NOVP y aún permanece en RC por 25 meses. El tercer caso (III-SA celularidad mixta) fue tratado con radioterapia y ABVD y está en RC por siete meses. El caso (IV-B), con depleción linfocitaria, sobrevivió dos meses y no recibió terapia.

Con respecto al tratamiento del VIH asociado a linfoma, la tendencia actual es utilizar el TARV en combinación con esquema CHOP con dosis modificada o completa ${ }^{26}$. En nuestra experiencia, cuatro pacientes recibieron, simultáneamente, TARV y tratamiento de su linfoma; tres de ellos están en RC y vivos.

\section{Conclusiones}

La incidencia acumulada de la asociación linfomainfección por VIH entre los años 1990 y 2002, fue de $0,68 \%$ en nuestra serie, por debajo del rango internacional, e incluso es menor a la referida en estudios nacionales previos.

La presencia de adenopatías es el signo clínico más constante en los pacientes estudiados. Por tanto, frente a pacientes VIH con adenopatías, de diámetro superior a 1,5 cm, de curso progresivo, con aumento de consistencia, fijación a tejidos, y persistencia en el tiempo, sin causa específica conocida, debería considerarse siempre efectuar la biopsia ganglionar. Además, en las recomendaciones para el manejo de la infección por $\mathrm{VIH}^{30}$, la presencia de adenopatías y fiebre encierra una fuerte posibilidad de linfoma. Es más, consideramos, como lo señalaran publicaciones al inicio de la epidemia en $1983^{28,29}$, que en cualquier etapa del SIDA, la biopsia ganglionar quirúrgica y la histopatología con un patrón atípico de proliferación linfoide debiera considerarse como un predictor útil del desarrollo de un linfoma. En el resto de las características clínico patológicas, la casuística presentada no difiere mayormente de lo señalado por autores foráneos.

Los resultados de este estudio indican, aunque la muestra es reducida, el pobre pronóstico de esta asociación y que existe un subgrupo de LNH y LH con 
mayores supervivencias, si el diagnóstico del linfoma se efectúa en estadios tempranos.

Los linfomas asociados con infección por VIH requieren, a escala nacional, una atención preferencial. Debiera crearse un Grupo Cooperativo Chileno que tenga como objetivos mantener un registro actualizado de estos pacientes; realizar estudios multicéntricos prospectivos, utilizar el IPI (International Prognostic Index) score $^{30}$, esquemas de quimioterapia agresivos con empleo simultáneo de TARV, instauración sistemática de profilaxis de las infecciones oportunistas y mantener permanentemente informada a la comunidad, del resultado de las investigaciones.

\section{Resumen}

Se revisó la asociación de linfoma e infección por VIH en un complejo hospitalario de la Región Metropolitana Sur de Santiago de Chile, su incidencia, características clínicas y patológicas, terapia y evolución en
14 casos. La incidencia acumulada (enero 1990 y diciembre 2002) fue de $0,68 \%$. Diez pacientes tenían linfoma no Hodgkin (siete de alto grado de malignidad y $50 \%$ en estadios III-IVB) y cuatro Hodgkin (tres con celularidad mixta, dos en estadio IVB). Diez pacientes con linfoma estaban en etapa C3 de SIDA según criterios del CDC, con un promedio de CD4 de 139 células/ $\mathrm{mm}^{3}$ y carga viral de 212.600 copias de ARN/ml. Ochenta y seis por ciento tenía afección ganglionar, localizada o generalizada. El tratamiento de los linfomas no Hodgkin de alto grado y extendidos fue con quimioterapia $\mathrm{CHOP}$ convencional, y en los linfomas de Hodgkin avanzados se aplicó el esquema ABVD. Seis pacientes recibieron tratamiento antiretroviral, cuatro simultáneamente con quimioterapia. La mortalidad global fue de $71 \%$, por progresión tumoral y/o sepsis. Cuatro pacientes sobreviven (18 a 50 meses) en remisión completa, dos linfomas no Hodgkin y dos Hodgkin. Se discuten la baja incidencia de la asociación linfoma y SIDA y la mayor frecuencia de linfomas con adenopatías localizadas o generalizadas.

\section{Referencias}

1.- Wolff M R, Northland R A, Segovia J P, Beltrán C B, Valdés M M, Albornoz M D, et al. Características clínicas e historia natural de la infección por virus de la inmunodeficiencia humana. Estudio en una población chilena atendida en un centro piloto multiprofesional. Rev Méd Chile 1995; 123: 61-73.

2.- Wolff M R, Diomedi A A, Morales O B, Bidart T H, Dabanch J P, Bustamante C M, et al. Seguimiento prospectivo de una población infectada por VIH con y sin posibilidades de terapia anti-retroviral: impacto en sobrevida y complicaciones. Rev Méd Chile 2001; 129: 886-94.

3.- Boletín epidemiológico CONASIDA MINSAL Chile, 2004.

4.- The non-Hodgkin's lymphomas pathologic classification project. National Cancer Institute sponsored study of classifications of non-Hodgkin's lymphomas. Cancer 1982; 49: 2112-35.

5.- Carbone P P, Kaplan H S, Musshoff K, Smithers D W, Tubiana M. Report of the Committee on Hodgkin's disease staging classification. Cancer Res 1971; 31: 1860-1

6.- Beltrán C B. Departamento de Infectología. Servicio de Medicina. Complejo Hospitalario Barros Luco. (Comunicación personal).

7.- Navarro J-T, Ribera J-M, Oriol A, Vaquero M, Romeu J, Batlle M, et al. International Prognostic Index is the best prognostic factor for survival in patients with AIDS-related non-Hodgkin's lymphoma treated with CHOP. A multivariated study of 46 patients. Haematologica 1998; 83: 508-13.

8.- Solal-Céligny P, Brousse N. Non Hodgkin's lymphomas associated with human inmunodefiency virus (HIV) infection. Solal-Céligny. Editors. Non Hogkins's Lymphomas. 3a edición Londres: Masson Publishing; 1993: 161-7.

9.- International Collaboration on HIV and Cancer. Highly active antiretroviral therapy and incidence of cancer in human immunodeficiency virus-infected adults. J Natl Cancer Inst 2000; 92: 1823-30.

10.- Biggar R J. Cancer in acquired immunodeficiency syndrome: An epidemiological assessment. Semin Oncol 1990; 17: 251-60.

11.- Ziegler J, Beckstedd J, Volberding P A, Abrams D I, Levine A M, Lukes RJ, et al. Non Hodgkin's lymphoma in 90 homosexual men. N Engl J Med 1984; 311: 565-70.

12.- Knowles D M, Chamulak G A, Subar M, Burke J S, Dugan M, Wernz J, et al. Lymphoid neoplasia associated with the acquired immunodeficiency syndrome (AIDS): The New York University Medical Center experience with 105 patients (19811986). Ann Intern Med 1988; 108: 744-53.

13. - Buela-Casal G, Bermúdez M P, Sánchez A I G, De los Santos- Roig M. Situación del VIH/ SIDA en Latinoamérica al final del siglo XX. Análisis de las diferencias entre países.
Rev Méd Chile 2001; 129: 944-54.

14.- Wolff M R. Cambios epidemiológicos en las enfermedades infecciosas en Chile durante la década 1990-2000. Rev Méd Chile 2002; 130: 353-62.

15.- Spivak J L, Bender B S, Uinn T C. Hematologic abnormalities in the acquired immune deficiency syndrome. Am J Med 1984; 77: 224-28.

16.- Fodor M. Cáncer y SIDA. En: Sepúlveda CC, Afani AS editores. SIDA. $3^{\mathrm{a}}$ edición Santiago: Editorial Mediterráneo; 2002: 176-205.

17. - Torrealba G M, Acuña G L, Tagle P M, Tapia J I, Huete I L. Valor de la biopsia cerebral en pacientes con SIDA y lesiones expansivas cerebrales. Rev Méd Chile 1990; 118: 1367-71.

18.- Chernilo S S, Trujillo S V, Meneses M C, Ríos J A, Aguirre J J. Compromiso pulmonar por linfoma no Hodgkin diseminado en un paciente con SIDA. Rev Méd Chile 2001; 129: 1315-9.

19.- Dolcetti R, Boiocchi M, Gloghini A, Carbone A. Pathogenic and histogenic features of HIV-associated Hodgkin's disease. Eur Cancer 2001; 37: 1276-87.

20.- Re A, Casari S, Cattaneo C, Facchetti F, Cadeo G, Carosi G, et al. Hodgkin's disease developing in patients infected by human immunodeficiency virus results in clinical features and prognosis similar to those in patients with human immunodeficiency virus related non- Hodgkin's lymphoma. Cancer 2001; 92: 2739-45. 
21.- Levine A M. Acquired immunodeficiency syndrome-related lymphoma: clinical aspects. Semin Oncol 2000; 27: 442-53.

22.- Calderero V A, Segura A H, Díaz R B, Aparicio J U, Navarro V I, Gómez J C. Primitive non Hodgkin's lymphoma of the pancreas in a patient with HIV infection. Rev Oncol 2003; 5: 223-5.

23.- Sparano J A. Clinical aspects and management of AIDS-related lymphoma. Eur J Cancer 2001; 37: 1296-305.

24.- PANDA. Protocolos Nacionales, Programa de Drogas Antineoplásicas del Adulto 20002001, Unidad de Cáncer, Ministerio de Salud, Chile, p 29.
25.- Kaplan L D, Straus D J, Testa M A, von Roenn J, Dezube B J, Cooley T P, et al. Low- dose compared with standard-dose $\mathrm{m}$ BACOD chemotherapy for non-Hodgkin's lymphoma associated with human immunodeficiency virus infection. N Engl J Med 1997; 336: 1641-8.

26.- Tam H K, Zhang Z F, Jacobson L P, Margolick J B, Chmiel J S, Rinaldo C, et al. Effect of highly active antiretroviral therapy on survival among HIV-infected men with Kaposi sarcoma or non- Hodgkin's lymphoma. Int J Cancer 2002; 98: 916-22.

27.- Wolff M, Northland R. Infección por VIH: recomendaciones para el manejo del adulto asintomático y de algunos síndromes clínicos. Rev Méd Chile 1996; 124: 983-98.

28.- Brynes R K, Chan W C, Spira T J, Swing E $\mathrm{P}$, Chandler FW. Value of lymph node biopsy in unexplained lymphadenopathy in homosexual men. JAMA 1983; 250: 13137.

29.- Ioachim H L, Lernes C W, Tapper M L. Lymphadenopathies in homosexual men. JAMA 1983; 250: 1306-9.

30.- Segura A H, López T P, Romera B B, Yuste A L I, Girones R S, Pérez J A F, et al. Non- Hodgkin's lymphomas with systemic presentation in patients with HIV infection. Rev Clin Esp 2004; 204: 303-7. 